\title{
Les vidéos d'exemples de pratique pour susciter le changement
}

\section{Florian Meyer}

\section{(2) OpenEdition}

Édition électronique

URL : http://journals.openedition.org/ripes/660

ISSN : 2076-8427

Éditeur

Association internationale de pédagogie universitaire

Référence électronique

Florian Meyer, «Les vidéos d'exemples de pratique pour susciter le changement », Revue internationale de pédagogie de l'enseignement supérieur [En ligne], 28(2) | 2012, mis en ligne le 06 novembre 2012, consulté le 07 septembre 2020. URL : http://journals.openedition.org/ripes/660

Ce document a été généré automatiquement le 7 septembre 2020.

Article L.111-1 du Code de la propriété intellectuelle. 


\title{
Les vidéos d'exemples de pratique pour susciter le changement
}

\author{
Florian Meyer
}

\section{Introduction et problématique}

1 Comme de nombreux pays, le Québec s'est engagé, depuis le début des années 2000, dans une réforme de l'éducation qui se caractérise par une refonte des programmes de formation des jeunes Québécois (Gouvernement du Québec, 2001). Cette réforme est le fruit d'un vaste processus de consultation sur l'état de l'éducation au Québec. Dans le cadre de cette réforme appelée "renouveau pédagogique", plusieurs modifications sont apportées et demandent aux enseignants de changer de façon significative leurs pratiques.

2 Une caractéristique majeure de ce programme réside dans le fait qu'il s'agit d'un programme par compétences. En effet, il n'est plus formulé en termes d'objectifs à atteindre, comme le programme précédent, mais en termes de compétences à développer. Ceci modifie considérablement les orientations en matière d'apprentissage et d'enseignement. L'intégration des matières et le développement de compétences transversales caractérisent également la réforme scolaire québécoise (Gouvernement du Québec, 2001). Les élèves doivent ainsi apprendre à extraire de leurs différentes matières des stratégies plus générales - telles la sélection d'informations appropriées, la résolution de problèmes ou une communication efficace - qui traversent tous les domaines d'apprentissage.

3 La réussite d'une telle réforme repose en grande partie sur une formation du personnel enseignant appropriée et cohérente avec les nouvelles exigences. Le Ministère de l'Éducation du Loisir et du Sport du Québec (MELS) a donc choisi de créer un référentiel de compétences professionnelles définissant non seulement les compétences attendues des enseignants, mais aussi les orientations générales de formation et les profils de sortie au terme de la formation initiale à l'enseignement (Gouvernement du Québec, 1999 ; Martinet, Raymond \& Gauthier, 2001). Ce référentiel décrit douze compétences 
professionnelles qui couvrent les différentes dimensions de l'agir enseignant: les compétences relatives aux fondements de l'enseignement en tant que profession, les compétences relatives à l'acte d'enseigner, les compétences relatives au contexte social et scolaire, et enfin, les compétences relatives à l'identité professionnelle (Martinet et al., 2001).

4 Avant de débuter cette recherche, nous avons rencontré des conseillers pédagogiques qui s'entendaient alors pour dire que la charge demandée aux enseignants en exercice était très lourde et que ceux-ci avaient d'importants besoins de support et de formation. En effet, la modification des pratiques est un processus complexe et long qui demande un investissement considérable de la part des enseignants (Archambault \& Richer, 2002 ; Darling-Hammond, 2000a ; Perrenoud, 1999). Or, le support offert ne semble pas répondre à ces besoins. Par conséquent, même si le renouveau pédagogique est mis en application depuis 2001, les enseignants n'ont pas toujours eu l'opportunité de développer suffisamment les compétences professionnelles nécessaires, et n'ont pas disposé nécessairement des ressources pour le faire. Le Québec se trouve, par conséquent, dans une situation où les enseignants, et la profession vers laquelle ils tendent, ont besoin de progresser rapidement et dans laquelle il est important de développer de nouveaux modèles de développement professionnel afin de répondre efficacement aux besoins du personnel enseignant.

Un dispositif de formation innovant appelé «Zoom sur l'expertise pédagogique » a été développé dans le but de proposer de nouvelles stratégies de formation pour les enseignants québécois. S'appuyant sur plusieurs travaux démontrant que l'utilisation de vidéos d'exemples de pratiques représente une opportunité intéressante pouvant répondre à ces besoins de formation (Ertmer, Conklin \& Lewandowski, 2002 ; Faingold, 1997 ; Mottet, 1997 ; Plakhotnik, 2001), ce dispositif multimédia dynamique en ligne intègre des fonctionnalités permettant de créer des activités de formation exploitant des exemples de pratiques sur vidéo (Meyer, 2010). Or, bien qu'un engouement certain soit exprimé pour de tels dispositifs un peu partout dans le monde (Meyer, soumis), aucune recherche ne permettait alors de déterminer l'effet de leur utilisation sur le développement professionnel des enseignants et, plus particulièrement, sur le changement de leurs pratiques.

Compte tenu de ce contexte particulier de renouveau pédagogique et des besoins spécifiques qu'il génère chez les enseignants québécois, il semblait donc nécessaire et pertinent de documenter, d'analyser et de comprendre l'effet que peut avoir l'utilisation de vidéos d'exemples de pratiques sur le développement professionnel des enseignants québécois en formation continue.

\section{Cadre théorique}

$7 \quad$ Le cadre théorique de cette recherche s'articule autour de quatre notions principales. Tout d'abord, la notion de développement de compétences professionnelles qui nous permet de comprendre dans quel processus les participants s'inscrivaient. Ensuite, la notion de dispositif de formation en ligne intégrant des vidéos afin de bien comprendre de quel dispositif de formation il était question dans cette recherche. Et enfin, les concepts de changement de pratique et de sentiment d'auto-efficacité qui permettent de délimiter le modèle d'analyse et de préciser la question de recherche. 


\subsection{Le développement des compétences professionnelles}

De par sa nature même, une profession est en permanente évolution. Par ailleurs, les contextes dans lesquels les professionnels de l'enseignement doivent agir sont toujours uniques (Perrenoud, 1996). Par conséquent, ces professionnels doivent s'assurer que leurs connaissances et leurs compétences sont toujours pertinentes afin de pouvoir agir le plus efficacement possible, quelles que soient les nouvelles situations dans lesquelles ils se retrouvent. Le terme de « développement professionnel » qualifie le processus par lequel les professionnels mettent à jour leur pratique. Plusieurs notions importantes sont regroupées sous ce terme: le développement de compétences professionnelles (Darling-Hammond, 1999), le développement d'une attitude professionnelle (Perrenoud, 1996) et la responsabilité des professionnels en rapport avec leurs compétences et leur rôle (Martinet et al., 2001).

9 Les compétences professionnelles sont donc au cœur du processus de développement professionnel. Or, une compétence n'existe que par rapport aux situations dans lesquelles elle se manifeste. Le Boterf (2000) fait d'ailleurs la distinction entre la compétence réelle et la compétence requise. Cette distinction peut s'illustrer très facilement dans le cas de l'enseignement québécois : les compétences requises sont les compétences qu'énumère le référentiel du MELS et qui doivent être maîtrisées par tous les enseignants (Martinet et al., 2001) ; les compétences réelles sont révélées lors des actions menées par un enseignant dans une situation particulière. Les stratégies de développement professionnel doivent donc non seulement permettre aux professionnels la construction de connaissances théoriques qu'il est indispensable de posséder pour être en mesure de reconnaître une situation, un contexte ou un problème et d'y associer les ressources utiles lors de l'action, mais également de se préparer à utiliser les ressources spécifiques appropriées aux situations éventuelles (Le Boterf, 2000) et les transférer dans d'autres situations.

10 Par ailleurs, la motivation personnelle, la qualité de l'enseignement offert (É. Charlier, 1998), l'actualisation équitable des compétences et des connaissances de ces professionnels (Fullan \& Hargreaves, 1992), les résultats des élèves (Darling-Hammond, 2000b), le progrès des connaissances en éducation et la cohérence professionnelle de l'enseignement dépendent directement $\mathrm{du}$ développement professionnel des enseignants (Meyer, 2010). "Students' achievement levels are positively affected by teachers who are satisfied, motivated, experienced, and knowledgeable, and who remain to become a part of the school and community culture » (Russell \& McPherson, 2001, p. 10). Dans ce contexte, il est intéressant de s'interroger sur le rôle que peuvent jouer les exemples de pratiques sur vidéo dans le processus de développement des compétences professionnelles. Il est à noter que dans le cadre de cette recherche, nous nous sommes concentré sur la compétence professionnelle 4 du référentiel publié par le MELS : "Piloter des situations d'enseignement-apprentissage pour les contenus à faire apprendre, et ce, en fonction des élèves concernés et du développement des compétences visées dans le programme de formation » (Martinet et al., 2001, p. 85). 


\subsection{Qu'est-ce qu'un dispositif de formation en ligne intégrant des vidéos?}

11 Pour être en mesure de comprendre le rôle que peuvent jouer les exemples de pratiques sur vidéo dans un processus de développement professionnel, il est important de comprendre en quoi consiste le dispositif de formation grâce auquel les enseignants ont l'occasion de participer à des activités de formation intégrant des vidéos.

12 Le dispositif «Zoom sur l'expertise pédagogique» s'adresse aux formateurs ainsi qu'aux enseignants. Il est composé de plusieurs sections. Chacune d'entre elles présente une diversité d'options qui répondent aux intérêts et aux objectifs des différents utilisateurs. La première section du dispositif est appelée " Médiathèque ». Il s'agit d'un système d'indexation qui offre la possibilité d'effectuer des recherches dans la banque de vidéo et de sélectionner des séquences sur la base de critères multiples : domaine d'apprentissage, compétence professionnelle enseignante, approche pédagogique et niveau scolaire, notamment. La seconde section est intitulée « Activités de formation ». Elle comprend tous les parcours de formation publics et ceux auxquels sont inscrits les enseignants en formation. La troisième section est appelée "Coin des formateurs » et s'adresse principalement, comme son nom l'indique, aux formateurs d'enseignants. Dans cette section, il est possible de sélectionner des vidéos, de leur ajouter des commentaires dynamiques et de construire des parcours de formation.

«Zoom sur l'expertise pédagogique » est avant tout un dispositif de formation, c'est-àdire un contexte particulier grâce auquel peuvent se vivre différentes situations de formation. Ces situations relèvent non seulement des éléments mis à la disposition des apprenants, mais également des apprenants eux-mêmes et des fondements pédagogiques propres à l'institution à l'origine du dispositif (Bernard, 1999). L'apprenant est bien entendu celui pour qui le dispositif est créé, mais il est aussi celui qui détermine l'utilisation qu'il en fera en fonction de ses propres besoins et intérêts (Projet Polifemo, 2001). Retenons également qu'un dispositif représente aussi un cadre organisé dans lequel des prestations et des ressources (Paquelin, 2000) sont offertes aux apprenants afin de les soutenir dans leur démarche personnelle de formation.

Selon Charlier et Peraya (2003), les dispositifs technologiques présentent des caractéristiques spécifiques: intégration de médias divers, exploitation d'outils de communication particuliers, utilisation du réseau Internet, exploitation des ressources qui abondent sur le Web, création d'applications pédagogiques originales, etc. Souvent, les dispositifs technologiques offrent des outils de gestion qui permettent à chaque utilisateur de se retrouver dans un environnement personnalisé. Des outils de collaboration et de communication sont souvent exploités dans les dispositifs en ligne.

Notons bien sûr qu'un dispositif technologique n'est pas forcément un dispositif en ligne. De nombreux dispositifs technologiques de formation existants n'exploitent pas obligatoirement les technologies du réseau Internet (Paquette, 2002). Bernard (1999), quant à lui, affirme que les technologies et Internet jouent un rôle fondamental dans la formation en réduisant les distances physiques, en atténuant les écarts culturels et en respectant les rythmes et les besoins de chacun tout en offrant des occasions de partage et d'échanges simples. 
16 Nous pouvons dire que «Zoom sur l'expertise pédagogique » est à la fois un dispositif de formation et un dispositif technologique. Plus précisément, il s'agit d'un dispositif de formation en ligne destiné au personnel enseignant. S'il possède bon nombre des caractéristiques que nous venons de décrire, il en est une en particulier qui lui confère son originalité : l'utilisation du média vidéo.

17 Ce dispositif offre des options de développement pédagogique originales aux formateurs, dans le but de soutenir la création de nouvelles activités de formation facilitant la compréhension des fondements du renouveau pédagogique. Il est, en effet, possible de lier de multiples façons des contenus théoriques complexes avec des vidéos offrant des illustrations concrètes de leur mise en application dans la pratique. À l'instar de certains dispositifs (InTime, VisionQuest, VideoClassrooom) dont les concepteurs se sont inspirés, il est possible d'ajouter aux vidéos des commentaires dynamiques et des transcriptions simultanées du texte. Ces spécificités enrichissent l'exploitation du média vidéo en soutenant la compréhension et la réflexion des enseignants en formation. De plus, les enseignants éloignés peuvent ainsi bénéficier du regard d'experts à qui ils auraient peut-être difficilement accès autrement.

Les concepteurs ont également fait le choix de réaliser des vidéos présentant à la fois des entrevues d'élèves, des entrevues d'enseignants et des situations de classe réelles. Afin d'apporter un éclairage sur la vidéo visionnée, un certain nombre d'informations sont associées aux différentes séquences: description du contexte, résumé de la situation, compétences observées, etc.

19 L'intention est également de permettre aux formateurs d'adapter les formations qu'ils créent aux enseignants à qui ils s'adressent, en tenant compte des contextes particuliers dans lesquels ils se trouvent. Les activités de formations créées à l'aide du dispositif sont appelées parcours de formation. Grâce à ces parcours, les formateurs peuvent inciter les participants à réfléchir sur ce qu'ils observent, savent, souhaitent faire ou font. Un parcours correspond à une série d'étapes que les enseignants doivent réaliser pour développer progressivement une ou plusieurs compétences professionnelles ciblées. Chaque étape est représentée par une page Web comportant automatiquement un titre, un numéro d'étape, une amorce (sorte d'introduction à l'étape) et différentes sections organisant le contenu de l'étape (vidéos, questionnements, ressources variées, ou encore des commentaires précis à des moments opportuns insérés dans les vidéos). Dans le cadre de cette recherche évaluative, nous nous sommes concentré sur un parcours en particulier que quelques enseignants ont eu l'occasion de suivre. Nous y revenons un peu plus loin.

\subsection{Le concept de changement de pratique enseignante}

20 Afin de documenter, d'analyser et de comprendre l'effet que peut avoir l'utilisation d'un dispositif de formation intégrant des vidéos d'exemples de pratiques sur le développement professionnel du personnel enseignant québécois, notre recherche s'appuie sur un modèle d'analyse prenant en compte différents types de variables interagissant lors d'une situation de formation et intégrant l'idée de changement de pratique (B. Charlier, 1998). Ce modèle est représenté schématiquement dans la figure 1. 
Figure 1 : Modèle d'analyse d'après B. Charlier, 1998, p. 259

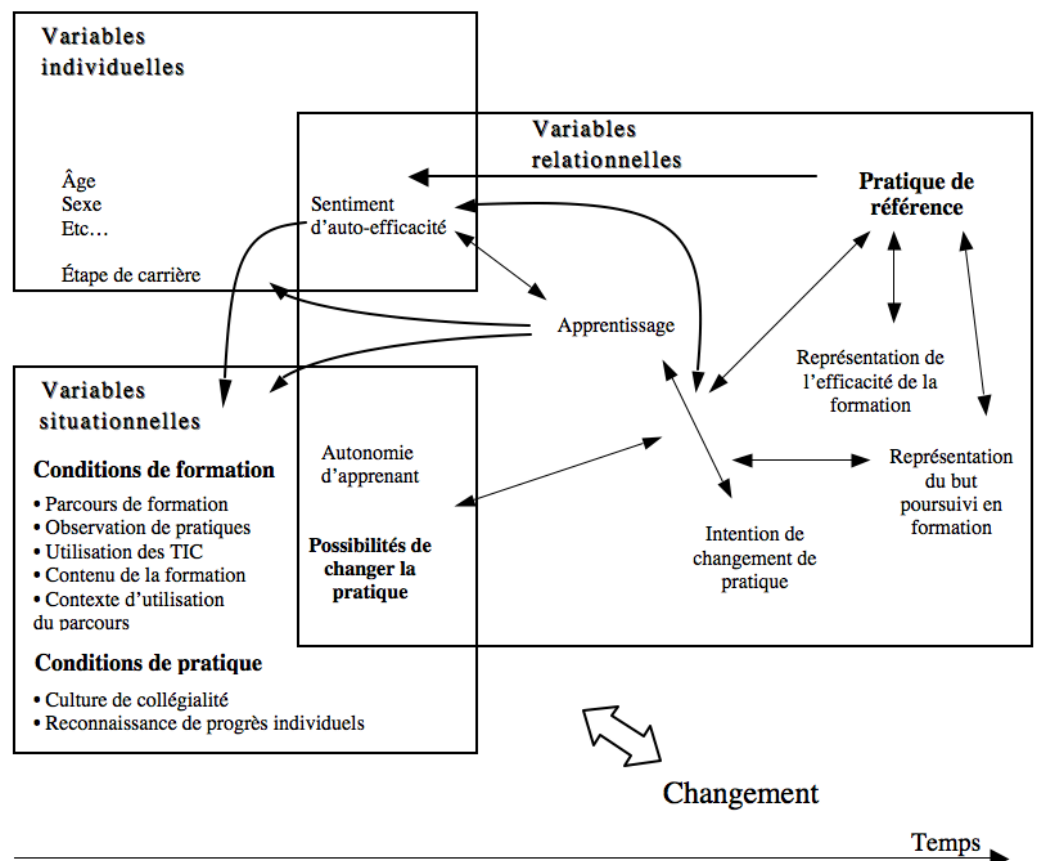

21 Les variables individuelles concernent les variables liées à la personne enseignante en formation uniquement: sexe, âge, étape de carrière et perception de sa capacité à enseigner (sentiment d'auto-efficacité (Bandura, 1994)). Les variables situationnelles concernent les variables en lien avec la situation de formation ou la situation de pratique de l'enseignant en formation. Les vidéos exploitées, le parcours de formation créé et l'utilisation du dispositif « Zoom sur l'expertise pédagogique » font partie de ces variables, par exemple. Enfin, les variables relationnelles concernent les variables issues de la relation entre la personne en formation et sa situation de formation et de pratique. Parmi ces variables se trouvent bien sûr l'apprentissage et les intentions de changement de pratique.

22 Nous avons déjà montré l'effet positif que peut avoir un parcours de formation intégrant des vidéos sur les apprentissages des enseignants, en particulier sur la connaissance des différentes composantes d'une compétence professionnelle (Meyer, soumis). Or, les objets d'apprentissage en formation des maîtres s'articulent autour des savoirs qui permettent aux enseignants de disposer de nouvelles ressources pour agir dans la pratique, autrement dit, d'être plus compétents (Le Boterf, 2000 ; Perrenoud, 1998). Notre recension nous a permis de constater trois types de liens entre l'apprentissage et le changement de pratique: le changement peut être inclus dans l'apprentissage, générateur d'apprentissage ou conséquence de l'apprentissage.

L'apprentissage peut être source de changement. Ceci signifie que le développement de nouvelles connaissances est un préalable au changement. Il s'agit donc de construire des savoirs variés qui vont ensuite être exploités pour changer ou simplement améliorer la pratique. Certains auteurs, à l'instar de Gabrielli (1989), parlent alors de transfert: "l'apprentissage ne consiste pas tant à maîtriser des connaissances et des capacités en situation "expérimentale" fermée qu'à les transposer in situ, dans les pratiques professionnelles, dans la perspective d'un changement de comportement ou d'attitude pédagogique» (Gabrielli, 1989, p.33). Knowles, de son côté, reprend le modèle de Tough (Knowles, 1990, p. 49). Ce modèle explique les relations entre les 
différents bénéfices qu'un apprenant adulte peut retirer d'une situation d'apprentissage. Il montre qu'en premier lieu, l'apprenant construit de nouveaux savoirs et de nouvelles habiletés. Ensuite seulement, ces nouveaux savoirs et habiletés sont exploités afin d'améliorer la pratique et les actions professionnelles. Pour ces auteurs, la construction de nouvelles connaissances est donc préalable au changement. D'autres auteurs estiment que le changement est générateur d'apprentissage. Mandeville (2002) a réalisé une recherche au cours de laquelle elle a rencontré un groupe d'étudiants en enseignement ayant réalisé des apprentissages importants à la suite d'expériences problématiques qui les ont amenés à changer leurs comportements ou leurs actions courantes. Elle a ainsi montré qu'« une situation problématique peut devenir le déclencheur d'un changement» (p. 222) et que ce changement amenait ces étudiants à un apprentissage qu'ils n'étaient pas prêts à vivre auparavant. Mandeville souligne que tous les sujets ayant vécu ce genre de situations manifestaient une certaine ouverture à vivre un bouleversement, et que l'apprentissage en était d'autant plus significatif.

Enfin, une grande proportion des chercheurs conçoit une interrelation plus dynamique et des allers et retours plus fréquents entre l'apprentissage et le changement de pratique. Des auteurs comme Brown et Duguid (1991) voient un lien étroit entre l'apprentissage et le changement de pratique : pour eux, le premier ne va pas sans le second et vice-versa. Ils encouragent même la création de formations plus articulées à la pratique, car cela renforcera encore ces liens qui assurent une meilleure qualité de pratique. En évoquant les modèles de Korthagen (1988), de Shulman (1986) et de Bennet et Carre (1993), qui intègrent tous le changement de pratique dans l'apprentissage, B. Charlier (1998) souligne le lien étroit entre les deux processus. Schön (1994), quant à lui, parlait de l'activité réflexive de l'enseignant qui questionne sa pratique et ses actions afin de les améliorer ce qui débouche sur la construction de nouveaux savoirs pratiques. Nous retenons donc que l'apprentissage et le changement de pratique sont mutuellement influents et que l'influence est plus ou moins forte dans un sens ou dans l'autre selon les situations et les personnes. Le changement engendre le développement de nouvelles connaissances et les nouvelles connaissances permettent l'innovation et le changement. B. Charlier (1998) précise un peu mieux cette idée : "Le changement de pratique d'enseignement concernerait un changement de décision de planification, un changement d'intention impliquant des modifications à d'autres niveaux de la pratique (quoi ?). Ce changement résulterait d'une réflexion sur l'action (comment ?), soit d'une réflexion technique (ça marche ou ça ne marche pas), soit d'une réflexion pratique (intégration des propres critères de l'enseignant pour développer une compréhension de sa pratique). Il serait assimilé au processus d'apprentissage de l'enseignement par l'action et la réflexion » (p. 78).

Un certain consensus se fait autour de la nécessité pour les praticiens de changer leurs pratiques afin de s'adapter aux exigences du renouveau pédagogique (Chouinard, 2002). Mais il est important de porter un regard attentif aux types de changement qui peuvent avoir effectivement lieu. En effet, les changements souhaités sont principalement des changements stables et fondamentaux, à ne pas confondre avec les changements ponctuels et techniques, plus facilement observables mais ne correspondant pas à la même construction de connaissances. À ce propos, inspirés des travaux de Watzlawick, Weakland et Fisch (1975), Bourgeois et Nizet (1997) évoquent deux niveaux de changement : le premier niveau " peut être observé lorsque le sujet modifie son point 
de vue initial sans remise en cause des prémisses conceptuelles ou théoriques de ce dernier» (p.92) et le second niveau, lorsque "le sujet accède à un cadre "métathéorique" nouveau, qui permet de dépasser, en les distinguant et les articulant de façon satisfaisante, les deux points de vue divergents de départ » (p. 94). Ces auteurs opposent donc ici deux types de changement, qui ne concernent pas les mêmes aspects des structures de connaissances. B. Charlier (1998) résume cette différence en affirmant que les changements de type 1 sont, en somme, "un petit peu plus de la même chose " (p. 88), alors que ceux de type 2 correspondent à « un changement de vision des choses, un recadrage » (p. 88). Ceci nous permet de bien comprendre que le changement peut se faire à différents niveaux et qu'il est important de bien les considérer pour comprendre le véritable effet que la formation mise en œuvre dans notre expérimentation peut avoir sur les intentions de changement de pratique. Ceci nous permet également de porter une attention particulière aux connaissances construites, qui, selon leur niveau d'abstraction, vont engendrer tantôt un changement de type 1 tantôt un changement de type 2 .

Nous venons de présenter différents aspects du concept de changement de pratique qui nous permettent de renforcer le lien interactif qui unit l'apprentissage, les intentions de changement de pratique et le changement de pratique. Enfin, nous retenons que les changements de pratique peuvent être de différents types et que ceux-ci sont fortement liés aux buts poursuivis durant la formation. Ces différents types s'expriment au travers des intentions de changement initiales qu'expriment les participants.

\subsection{Le concept de sentiment d'auto-efficacité}

28 Le sentiment d'auto-efficacité revêt un caractère spécial dans notre modèle d'analyse et il est important de le définir précisément. Le concept d'auto-efficacité (traduction du terme "self-efficacy») consiste en la croyance qu'une personne a en sa capacité à réaliser des tâches importantes de la vie avec un niveau de performance satisfaisant (Bandura, 1994). Bandura précise que ce sentiment influence le bien-être des personnes, leurs pensées, leur motivation ainsi que leurs comportements. Ainsi, les personnes possédant un fort sentiment d'auto-efficacité sont plus accomplies et se sentent mieux dans leur peau. Ces personnes font également preuve d'une plus grande tolérance face aux difficultés, sont plus motivées et relèvent des défis de plus grande ampleur. Elles sont également moins angoissées et moins vulnérables.

Le sentiment d'auto-efficacité découle de quatre sources différentes: des expériences réussies, l'expérience vicariante, la persuasion sociale et la prédisposition naturelle (Bandura, 1997). L'influence sur le sentiment d'auto-efficacité de chacune de ces quatre sources est différente et chacune d'entre elles peut avoir un effet négatif selon les contextes et les situations. La seconde source est celle qui nous intéresse le plus dans cette recherche. Il s'agit de l'expérience vicariante. Pour que l'influence de ce facteur soit déterminante, il faut que la personne qui observe perçoive des similitudes entre le modèle et elle-même. Si le modèle observé éprouve du succès, le sentiment d'autoefficacité de l'observateur sera influencé d'autant plus positivement que l'identification au modèle est grande. Réciproquement, si la personne observée échoue et que la personne observante se reconnaît en elle, alors le sentiment d'auto-efficacité sera fortement influencé négativement. 
30 Notre intérêt pour le sentiment d'auto-efficacité vient surtout de l'influence qu'il peut avoir sur les apprenants ou les praticiens. Dans une recension importante réalisée dans le but de mieux définir le sentiment d'auto-efficacité des enseignants, TschannenMoran et ses collègues (2001) ont relevé différentes études qui ont montré que les enseignants disposant d'un fort sentiment d'auto-efficacité faisaient preuve d'une plus grande persistance à aider les élèves en difficulté et d'une plus faible tendance à les critiquer pour se concentrer plutôt sur les stratégies de support. Les résultats de ces recherches révélaient également que ces enseignants offraient une plus grande variété de méthodes d'enseignement à leurs élèves, qu'ils étaient plus enclins à l'innovation et qu'ils cherchaient à améliorer leur pratique. En cohérence avec le modèle de Bandura (1997), les enseignants qui éprouvent un sentiment d'auto-efficacité élevé sont plus motivés, plus résistants aux difficultés, ils réalisent des choix plus appropriés et plus novateurs, ils sont plus efficaces et mieux concentrés sur leurs objectifs. Plus enthousiastes et positivement impliqués dans leur tâche, ils sont également moins sujets à la dépression.

31 Le rôle de ce sentiment dans les apprentissages et son caractère prédictif quant aux changements de pratique lui confère, dans notre modèle d'analyse, une place précise. B. Charlier (1998) parle dans son modèle de «vision de soi » et met en évidence les relations suivantes: les personnes qui se voient comme passives et vulnérables sont plutôt associées à une conception de l'apprentissage du type acquisition de connaissances, alors que les personnes qui se voient comme créatrices ou chercheuses sont plutôt associées à une conception de l'apprentissage de type construction de connaissances. Nous proposons d'associer les personnes ayant un fort sentiment d'auto-efficacité aux personnes se voyant comme des personnes créatrices et en confiance par rapport à leurs capacités, alors que les personnes vulnérables et passives seraient plutôt des personnes ayant un sentiment d'auto-efficacité plus faible. Nous remplaçons donc la «vision de soi " par le «sentiment d'auto-efficacité ». Le modèle d'auto-efficacité des enseignants présenté ci-dessus fournit des pistes d'interprétation plus précises pour comprendre les attitudes, les comportements et les apprentissages d'un enseignant dans une situation de développement professionnel. Nous devons donc considérer, dans la mesure du possible, ce sentiment d'auto-efficacité ainsi que ses conséquences au niveau de l'apprentissage ou des intentions de changement de pratique des personnes ayant participé à cette recherche.

L'objectif spécifique de cette recherche est donc de décrire les intentions de changement de pratique, en ce qui concerne la compétence «Piloter des situations d'enseignement-apprentissage pour les contenus à faire apprendre, et ce, en fonction des élèves concernés et du développement des compétences visées dans le programme de formation", des enseignantes et des enseignants ayant suivi un parcours de formation portant sur cette compétence et intégrant des vidéos d'exemples de pratique. Cette description a pour but de répondre à la question : quels ont été les effets du dispositif de formation sur les intentions de changement de pratique des enseignants qui l'ont utilisé?

\section{Méthodologie}

La recherche que nous avons réalisée est de type évaluation différée (Gabrielli, 1989). Nous nous sommes centré sur l'évolution de six personnes volontaires (cinq 
enseignantes et un enseignant, tous du primaire) ayant suivi un parcours de formation réalisé avec le dispositif en ce qui concerne leurs intentions de changement de pratique (Meyer, soumis).

Nous situant dans un paradigme plutôt interprétatif (Lessard-Hébert, Goyette \& Boutin, 1990), où la réalité est un construit subjectif dépendant des représentations des participants et du chercheur, nous avons pris en compte l'évolution de chaque individu concerné selon son point de vue et ses représentations en nous appuyant sur le modèle d'analyse pour identifier les multiples éléments interreliés en jeu dans la situation de formation évaluée.

La collecte de données se faisait, pour chaque personne, en trois étapes distinctes et réparties sur une période de un à deux mois débutant en décembre 2004.

1. Première étape: la première entrevue a été réalisée quelques jours avant que les participants ne débutent le parcours. Cette entrevue était individuelle et durait environ une heure. L'ordre des questions et des composantes abordées (cf. Annexe 1) n'était pas imposé aux participants. Toutefois, le canevas était attentivement respecté. Pour chaque bloc de questions lié à une composante, nous énoncions tout d'abord l'intitulé exact de la composante, puis nous présentions celle-ci sur une feuille de papier préalablement imprimée en gros caractères, afin que les participants puissent la relire aisément. Cette feuille de papier restait visible en permanence pendant que les participants parlaient de la composante. Quelques questions étaient parfois reformulées lorsque ils ne semblaient pas avoir compris la formulation initiale, mais ces reformulations ne contenaient aucune piste de réponse ni d'exemple. Dès que les participants souhaitaient aborder une autre composante, nous retirions la feuille et répétions le procédé pour la nouvelle composante ;

2. Seconde étape : suite à une brève présentation du dispositif et de ses caractéristiques, les participants ont suivi le parcours. Cette étape s'est déroulée sur une période variant entre quatre et six semaines selon la capacité des participants à dégager du temps pour cette formation. Durant cette période, aucun contact avec le chercheur n'était demandé, sauf quelques jours avant la fin du parcours afin de fixer un rendez-vous pour la seconde entrevue ;

3. Troisième étape : la seconde entrevue a été réalisée quelques jours (maximum une semaine) après que les participants eurent terminé le parcours. Elle se déroulait selon le canevas prévu et dans des conditions similaires à celles de la première entrevue.

L'analyse des données récoltées s'est faite en plusieurs étapes selon une démarche déductive (Lessard-Hébert et al., 1990) partant de notre modèle d'analyse. Tout d'abord, une retranscription des entrevues a été réalisée par une tierce personne ne connaissant ni les participants ni le sujet de la recherche. Chaque entrevue a fait l'objet d'un verbatim stocké dans un fichier informatique de type document texte. Ensuite, après avoir été lu et vérifié, chaque verbatim a été annoté une première fois sous forme de courts énoncés résumant les propos des participants. Cette première étape a permis de dégager de nombreux éléments composant les réponses et les commentaires des participants. Une seconde lecture a ensuite permis d'affiner les annotations et d'amorcer les regroupements selon les différentes questions de recherche et le modèle d'analyse préalablement construit. Une troisième lecture nous a finalement amené à affiner les regroupements en distinguant différentes dimensions de données. 


\section{Résultats} 1 résume cette dimension selon la légende suivante :

- Cap++ : Se sent très capable (ne voit pas d'obstacle)

- Cap+ : Se sent plutôt capable (voit quelques obstacles surmontables)

- Cap- : Ne se sent pas tellement capable (voit beaucoup d'obstacles)

- Cap-- : Ne se sent pas capable du tout (les obstacles sont insurmontables)

- Ess++ : Souhaite essayer de nouveaux aspects de cette composante

- Ess+ : Souhaite éventuellement essayer

- Ess = : Le fait déjà (le parcours conforte son expertise)

- Ess-- : Ne souhaite pas essayer du tout (ne fait pas mention de cette intention)

$\bullet_{+}^{+}$: Se sent plus à l'aise

- = : Pas de changement dans sa capacité

- - : Se sent moins à l'aise

- ?: Ne sait pas s'il y a changement

- nil : Aucune réponse

Tableau 1 : Synthèse de la dimension A

\begin{tabular}{|c|c|c|c|c|c|c|}
\hline & Sylvie & Hervé & France & Mireille & Martine & Chantal \\
\hline $\begin{array}{l}\text { C.1 } \\
\text { Entrevue } 1\end{array}$ & Cap + & Cap- & Cap + & Cap- & Сap+ & Саp+ \\
\hline
\end{tabular}




\begin{tabular}{|c|c|c|c|c|c|c|}
\hline $\begin{array}{l}\text { C. } 1 \\
\text { Entrevue } 2\end{array}$ & $\begin{array}{c}\text { Cap+ } \\
\text { Ess-- } \\
?\end{array}$ & $\begin{array}{l}\text { Cap+ } \\
\text { Ess++ } \\
=\end{array}$ & $\begin{array}{l}\text { Cap }+ \\
\text { Ess++ } \\
+\end{array}$ & $\begin{array}{l}\text { Cap+ } \\
\text { Ess-- } \\
=\end{array}$ & $\begin{array}{l}\text { Cap+ } \\
\text { Ess }= \\
=\end{array}$ & $\begin{array}{l}\text { Cap+ } \\
\text { Ess++ } \\
+\end{array}$ \\
\hline $\begin{array}{l}\text { C. } 2 \\
\text { Entrevue } 1\end{array}$ & Cap- & Cap++ & Cap-- & Cap- & Cap- & Cap ++ \\
\hline $\begin{array}{l}\text { C. } 2 \\
\text { Entrevue } 2\end{array}$ & $\begin{array}{l}\text { Cap- } \\
\text { Ess++ } \\
=\end{array}$ & $\begin{array}{l}\text { Cap++ } \\
\text { Ess++ } \\
+\end{array}$ & $\begin{array}{l}\text { Cap+ } \\
\text { Ess++ } \\
+\end{array}$ & $\begin{array}{l}\text { Cap+ } \\
\text { Ess++ } \\
+\end{array}$ & $\begin{array}{l}\text { Cap++ } \\
\text { Ess++ } \\
+\end{array}$ & $\begin{array}{l}\text { Cap++ } \\
\text { Ess-- } \\
=\end{array}$ \\
\hline $\begin{array}{l}\text { С. } 3 \\
\text { Entrevue } 1\end{array}$ & Cap + & Cap + & Cap + & Cap-- & Cap++ & Cap- \\
\hline $\begin{array}{l}\text { C. } 3 \\
\text { Entrevue } 2\end{array}$ & $\begin{array}{l}\text { Cap+ } \\
\text { Ess-- } \\
+\end{array}$ & $\begin{array}{l}\text { Cap+ } \\
\text { Ess++ } \\
=\end{array}$ & $\begin{array}{l}\text { Cap- } \\
\text { Ess++ } \\
+\end{array}$ & $\begin{array}{l}\text { Cap+ } \\
\text { Ess++ } \\
+\end{array}$ & $\begin{array}{l}\text { Cap+ } \\
\text { Ess+ } \\
=\end{array}$ & $\begin{array}{l}\text { Cap+ } \\
\text { Ess++ } \\
+\end{array}$ \\
\hline $\begin{array}{l}\text { C. } 4 \\
\text { Entrevue } 1\end{array}$ & Cap- & Cap- & Cap- & Cap + & Cap + & Cap ++ \\
\hline $\begin{array}{l}\text { C. } 4 \\
\text { Entrevue } 2\end{array}$ & $\begin{array}{l}\text { Cap+ } \\
\text { Ess+ } \\
=\end{array}$ & $\begin{array}{l}\text { Cap+ } \\
\text { Ess-- } \\
=\end{array}$ & $\begin{array}{l}\text { Cap-- } \\
\text { Ess+ } \\
=\end{array}$ & $\begin{array}{l}\text { Cap- } \\
\text { Ess-- } \\
=\end{array}$ & $\begin{array}{l}\text { Cap+ } \\
\text { Ess++ } \\
+\end{array}$ & $\begin{array}{l}\text { Cap+ } \\
\text { Ess++ } \\
-\end{array}$ \\
\hline $\begin{array}{l}\text { C. } \\
\text { Entrevue } 1\end{array}$ & Cap + & Cap + & Cap + & nil & Cap + & Cap + \\
\hline $\begin{array}{l}\text { C. } \\
\text { Entrevue } 2\end{array}$ & $\begin{array}{l}\text { Cap+ } \\
\text { Ess+ } \\
+\end{array}$ & $\begin{array}{l}\text { Cap+ } \\
\text { Ess++ } \\
=\end{array}$ & $\begin{array}{l}\text { Cap+ } \\
\text { Ess++ } \\
+\end{array}$ & $\begin{array}{l}\text { Cap+ } \\
\text { Ess++ } \\
+\end{array}$ & $\begin{array}{l}\text { Cap+ } \\
\text { Ess++ } \\
+\end{array}$ & $\begin{array}{l}\text { Cap+ } \\
\text { Ess++ } \\
+\end{array}$ \\
\hline
\end{tabular}

\subsection{Synthèse de la dimension $B$ : validation de la pratique}

41 La dimension B fait état du jugement des participants sur leur propre pratique et de la validation de leur pratique. Le type de validation est essentiellement descriptif, comme le démontre la synthèse suivante.

\subsubsection{Participante Sylvie}

Lors de la seconde entrevue, Sylvie porte un regard critique sur sa pratique. Elle constate notamment qu'elle était portée à donner les réponses et les informations aux élèves trop rapidement. En ce qui concerne la composante 2, elle pense qu'elle le faisait déjà bien mais qu'il y a de la place pour l'amélioration. Pour la composante 4, Sylvie constate qu'elle avait tendance à éviter assez facilement ces aspects.

43 Il est intéressant de constater que Sylvie affirme s'être beaucoup reconnue dans certaines vidéos : certains projets se rapprochent de ceux qu'elle fait avec ses élèves. 
Elle rappelle qu'elle préfère travailler avec des projets qu'avec des manuels et estime que cela fonctionne bien.

\subsubsection{Participant Hervé}

Hervé affirme que le parcours lui a permis de remettre beaucoup de choses en question. En prenant du recul durant le parcours, il a réalisé qu'il trouvait les situations présentées très intéressantes et qu'il n'avait pas fait cela depuis très longtemps avec ses élèves. Il souhaite retourner dans cette direction. Il a également réalisé qu'il pouvait mieux planifier et mieux organiser sa classe pour créer des conditions génératrices d'engagement. Il a réalisé qu'il pouvait pousser plus loin dans le questionnement, par exemple, ou dans la médiation. Il se sent influencé par ce qu'il a vu.

Hervé a réalisé qu'il mettait parfois un peu trop de ressources à disposition des élèves. Il a pris conscience qu'il doit sélectionner davantage et prendre plus de temps pour analyser et sélectionner les ressources pertinentes. Aussi, il a constaté qu'il pouvait améliorer sa pratique, être mieux organisé et donc plus détendu pour ainsi pouvoir mieux aider les élèves. Il a constaté que l'atmosphère était très importante et qu'il devait travailler là-dessus.

Hervé affirme qu'il aimerait améliorer son approche pour mieux travailler les démarches au niveau du groupe-classe, de la résolution de problème et du travail en équipe. Il sait également qu'il doit améliorer ses rétroactions. Il a toujours l'impression de ne pas aller assez en profondeur. Il fait de la rétroaction sur les tâches et les problèmes, mais pas assez sur les stratégies. Il pense qu'il comprend bien le transfert et qu'il soutient bien cela. Il constate également qu'il ne fait pas assez de retour sur les stratégies et devra en faire plus. Il a pris conscience qu'il est important de prendre plus de temps avec les élèves pour encadrer davantage les apprentissages. Enfin, il constate que les enseignants filmés sont de très bons modèles dont il souhaite s'inspirer car il sait qu'il a encore beaucoup à améliorer.

\subsubsection{Participante France}

Lors de la seconde entrevue, France valide sa pratique essentiellement en fonction de ce qu'elle a vu dans les vidéos. Elle a été notamment satisfaite de reconnaître des éléments de sa propre pratique dans les vidéos. Elle a constaté également qu'elle ne faisait pas assez de renforcement positif. Enfin, les vidéos lui ont permis de penser à des choses et à des façons de faire auxquelles elle ne pensait pas avant. Il lui reste à trouver une façon de faire qui lui convienne vraiment.

Le parcours lui a permis de constater qu'elle ne faisait pas assez appel à certaines ressources. Aussi, elle a réussi une activité en mathématiques qui utilisait des pailles. Elle pense que son activité était plus constructiviste et s'inscrivait dans ce qu'elle avait vu dans les vidéos. Elle était très satisfaite de son activité.

rappelle qu'elle en est encore, dans sa pratique en général, à un stade d'essaierreur et que si quelque chose ne fonctionne pas, elle le modifie et essaye à nouveau. Elle sait qu'elle doit essayer encore car elle ne réussit pas encore à le faire.

Enfin pour la compétence, elle était contente de constater qu'elle faisait plus de choses pertinentes qu'elle pensait. Elle a constaté qu'il y a d'autres choses qu'elle doit commencer à faire aussi. Elle sait de plus que son cas est particulier car elle n'a jamais 
commencé une année avec des élèves. Elle ne sait donc pas comment elle peut mettre en place un fonctionnement assurant l'autonomie des élèves.

Elle précise enfin que les vidéos lui ont permis de mieux comprendre ce qu'elle faisait et de savoir où elle se situait. Cependant, elle souligne qu'elle n'arrive pas encore à gérer la classe pour assurer l'autonomie de ses élèves. Elle n'a pas trouvé la recette magique. Donc elle ne peut pas trop mettre en application cette compétence. France trouvait d'ailleurs que parfois les vidéos ne donnaient pas de réponses assez précises sur la gestion de classe expliquant comment certaines situations étaient réalisables.

\subsubsection{Participante Mireille}

Durant la seconde entrevue, Mireille ne fait pas véritablement une validation de sa pratique. Elle fait principalement des commentaires sur ce qu'elle souhaite essayer ou améliorer. Elle reconnaît ainsi, en ce qui concerne la composante 1, qu'elle créait déjà des conditions pour que les élèves s'engagent. Seulement les concepts sont plus précis pour elle maintenant, elle pense donc qu'elle sera meilleure.

53 Elle souhaite ajuster le nombre de ressources qu'elle mettra à disposition des élèves et passera plus de temps avec les élèves pour mieux expliquer le fonctionnement des ressources. Elle souhaite également organiser plus de discussions en grand groupe et amener les élèves à mieux expliciter les stratégies qu'ils utilisent. En terminant par la compétence en général, elle précise vouloir être plus précise et mieux définir les activités qu'elle fera vivre à ses élèves.

\subsubsection{Participante Martine}

Le parcours a permis à Martine de constater qu'elle n'utilisait pas assez les échéanciers comme ressource. Elle a réalisé que ceux-ci sont très importants pour bien suivre l'évolution et le travail des élèves. Elle a également constaté qu'elle ne prend pas assez le temps de discuter avec les élèves pour les guider et les aider. Elle souhaite faire cela un petit peu plus.

Martine s'est rendue compte qu'elle ne faisait pas assez de questionnement et qu'elle escamote cela un peu trop souvent, mais elle a déjà essayé de commencer à le faire depuis le parcours. Ses essais l'ont d'ailleurs convaincue et elle se considère meilleure. Elle a constaté également, suite au parcours, qu'elle doit vraiment changer certaines choses dans sa pratique et qu'elle souhaite vivement le faire.

Enfin, elle pensait qu'elle était une personne organisée, mais elle constate en regardant les vidéos qu'elle devrait l'être plus. Elle a également réalisé qu'elle peut emmener les enfants encore plus loin.

\subsubsection{Participante Chantal}

57 Lors de la seconde entrevue, Chantal constate qu'elle ne fait pas assez souvent ce que cette composante préconise. Elle souhaiterait le faire beaucoup plus. Elle sent cette composante plus ancrée en elle maintenant. Elle se sent moins tentée de donner les réponses. Elle va faire plus travailler les élèves.

58 Concernant la seconde composante, elle confirme lors de la seconde entrevue qu'elle faisait déjà cela très bien. 
59 Relativement à la troisième composante, Chantal constate, lors de la première entrevue, qu'elle aide les élèves, mais ne sait pas si elle les guide suffisamment. Elle essaie, mais ne réussit pas toujours. Elle se sait meilleure en mathématiques qui est sa force. Lors de la seconde entrevue, Chantal souligne que, lors du parcours, elle a constaté, grâce aux vidéos, qu'elle avait tendance à donner les réponses aux élèves trop rapidement. Elle affirme qu'elle doit se débarrasser de vieilles habitudes de trop faire pour l'élève.

60 Lors de la seconde entrevue, Chantal affirme qu'elle oublie souvent de faire des rétroactions. Elle faisait cela beaucoup avant, revenir sur ce qu'ils avaient fait avant, mais elle oublie maintenant. Elle sait qu'elle devrait le faire plus.

61 Pour la compétence, lors de la première entrevue, Chantal rappelle qu'elle était en avance sur le programme. Elle constate que sa pratique a beaucoup changé grâce au renouveau pédagogique et qu'elle voit les résultats de ses changements : les enfants sont plus avancés. Elle considère que sa tâche est plus légère grâce au renouveau pédagogique et elle réussit à rendre les élèves beaucoup plus autonomes. Elle leur donne beaucoup plus et les rend responsables. Lors de la seconde entrevue, Chantal réalise qu'elle fait bien cette compétence, mais qu'elle ne sait pas jusqu'à quel point elle le fait bien. Les vidéos lui ont permis de réaliser également qu'elle donnait parfois trop rapidement les réponses aux élèves et qu'elle doit plus les amener à trouver ou réaliser par eux-mêmes.

62 En synthèse, nous pouvons retenir que les participants ont, dans l'ensemble, suite à l'observation des vidéos et du parcours, relevé des éléments leur rappelant leur propre pratique ce qui leur permet de la valider. Cependant, tous remettent en question certains aspects de leur pratique et définissent des pistes d'amélioration. Avant le parcours, aucun des participants ne porte un regard de validation sur sa pratique.

\section{Discussion}

63 L'analyse de ces dimensions nous donne l'occasion d'observer chez Sylvie une intention de changer sa pratique. Le parcours et les vidéos ont donc eu un véritable effet chez elle, mais celle-ci est essentiellement orientée vers des changements éventuels dans sa pratique plus que sur les apprentissages (Meyer, soumis). Nous pouvons donc nous demander si les changements qu'elle souhaite apporter seront suffisamment fondés pour permettre une amélioration en profondeur de sa pratique. D'autant plus qu'à plusieurs reprises, Sylvie mentionne que les vidéos l'ont découragée car elles présentaient des idéaux de pratiques difficiles à atteindre. Rappelons, par ailleurs, qu'il s'est produit un changement pouvant avoir une incidence importante sur le sentiment d'auto-efficacité de Sylvie. Lors de la première entrevue, elle mentionnait qu'elle était grandement reconnue dans ses progrès et ses compétences par ses collègues et sa direction; or, durant la seconde entrevue, elle souligne plutôt que la direction n'apprécie pas son style d'enseignement et qu'elle se sent remise en cause dans ses propres compétences. Cet aspect influence certainement la perception de Sylvie quant à ses propres compétences ainsi que sa volonté de prendre de nouveaux risques et d'apporter des changements de type 2 dans sa pratique.

De tous les participants, Hervé est le seul qui possède une expérience importante et une connaissance précise de la compétence et de ses composantes. Il est également l'un des 
rares - avec France - à faire preuve d'une véritable intention d'améliorer sa pratique pour chacune des composantes, en soulignant de plus le fait qu'il a encore beaucoup à apprendre et à améliorer. Ses connaissances antérieures et sa volonté d'analyser les pratiques pour en retirer le plus d'apprentissages possible ont permis à Hervé d'aller jusqu'à relever dans les vidéos certains aspects qui ne sont pas liés à la compétence ciblée par le parcours : l'organisation du groupe classe, par exemple. Le parcours a donc non seulement eu un effet sur ses connaissances et son intention de changer sa pratique en ce qui concerne la compétence ciblée, mais il lui a permis également d'envisager des pistes d'amélioration de sa pratique à d'autres égards.

Le cas de France est particulièrement concluant, parce que l'ensemble des variables que nous avons étudiées se conjuguaient toutes pour produire un effet majeur sur ses apprentissages et son intention de changement de pratique. En effet, en ce qui concerne les variables individuelles, France est jeune, en début de carrière et avide de nouvelles connaissances. Par ailleurs, le parcours a contribué à développer un fort sentiment d'auto-efficacité et à la rassurer quant à ses compétences et à son niveau de pratique. En ce qui concerne sa pratique de référence et ses représentations du but poursuivi ou de l'efficacité de la formation, France se plaçait dans une posture qui lui permettait d'envisager des intentions de changement de pratique importantes. En combinant à ces variables les apprentissages réalisés (Meyer, soumis), les conditions de formation efficaces, des conditions de pratiques collégiales et une reconnaissance de ses progrès par ses collègues, il était prévisible, selon notre modèle d'analyse, que les intentions de changements de pratique de France soient si marquées et importantes. Il serait maintenant intéressant de vérifier si les contraintes relatives à la gestion de classe dont elle faisait mention auront raison de ses intentions et l'empêcheront effectivement de changer sa pratique.

Même si Mireille estime n'avoir pas progressé en ce qui concerne la première composante et la quatrième, dans tous les cas, elle précise son intention d'essayer ce que le parcours lui a permis de réaliser ou de découvrir. Lorsqu'elle ne mentionne pas son intention d'essayer de nouveaux aspects, ce qui est le cas pour la composante 4, Mireille précise qu'elle souhaite se former et en apprendre encore plus sur la composante. Cela démontre une véritable intention de progresser et de s'améliorer. Le parcours et les vidéos ont permis à Mireille de constater que sa pratique est de qualité, ce qui l'encourage et la motive, mais ils lui ont également permis de reconnaître les aspects sur lesquels elle peut encore progresser et lui ont donné le désir de suivre d'autres formations ou d'observer ses collègues compétents. Si l'on combine ses intentions clairement formulées et un sentiment d'auto-efficacité grandement renforcé, associé à une perception positive de la formation qu'elle vient de suivre et de l'importance des apprentissages réalisés, notre modèle d'analyse nous porte à croire que Mireille se dirige vers des changements de pratique conséquents et approfondis. Le parcours et les vidéos qu'il contient ont amorcé des changements importants chez Mireille, qui fait preuve d'une plus grande confiance et d'une volonté évidente d'améliorer sa pratique à tous les niveaux. Cela nous porte à croire que la spécification, par Martine, d'intentions de formation auxquelles le parcours et ses vidéos ont su répondre, l'a orientée positivement vers une volonté de changer sa pratique.

67 Martine a profité des vidéos non seulement pour trouver de nouvelles pistes ou orientations à sa pratique, mais également pour se rassurer et confirmer que sa pratique est de bonne qualité et que tout ne doit pas obligatoirement être modifié. 
Cette prise de conscience se retrouve chez plusieurs des participants et joue un rôle positif dans tous les cas, car elle permet à ces personnes de ne pas se sentir menacées par ce qui est présenté, mais plutôt de se sentir compétentes tout en laissant de la place à l'amélioration. Cette amélioration du sentiment d'auto-efficacité, due à une expérience vicariante positive, permettra à Martine d'être plus résistante aux difficultés que le changement de sa pratique lui procurera, et surtout elle permettra à Martine d'oser davantage et de s'engager plus audacieusement dans des changements de type 2 .

Chantal souligne un aspect que France avait déjà relevé : le silence régnant dans les classes filmées. Chantal prétend ne pas arriver à obtenir un tel silence dans sa classe, ce qui représente un obstacle important et limite ses actions. Bien que Chantal affirme se sentir plus compétente suite au parcours, ces problèmes de gestion de classe restent des obstacles que les vidéos lui ont rappelés. Bien entendu, la compétence professionnelle ciblée dans le parcours implique de soutenir l'engagement des élèves et de faire en sorte que chacun soit actif dans ses apprentissages, ce qui, en conséquence, facilite la gestion de classe. Les vidéos avaient notamment pour objectif de mettre en évidence ce lien, mais Chantal ne semble pas l'avoir perçu. Elle semble plutôt avoir relevé un contraste important entre le silence des classes filmées et son propre contexte de pratique. Dans ce cas, les vidéos et la façon dont elles ont été utilisées semblent donc avoir eu un effet plutôt décourageant sur la volonté de Chantal de changer sa pratique. Ici, nous pouvons dire que la pratique de référence place la participante dans une posture qui ne lui permet pas de bénéficier positivement de l'exemple observé. L'attention de Chantal a été détournée vers un élément perturbateur ayant vraisemblablement un effet négatif sur ses intentions de changement de pratique. Malgré tout, Chantal formule certaines de ses intentions de changement de pratique comme des défis. Elle formule ainsi son envie d'améliorer sa pratique, mais également le fait qu'elle a conscience qu'il lui reste encore beaucoup de chemin à parcourir et qu'elle souhaite y aller étape par étape.

Par ailleurs, comme ce fut le cas pour d'autres participants, Chantal semble se sentir moins capable de réaliser ce que demande la composante 4 après le parcours qu'avant. Chantal souligne d'ailleurs à quel point il lui reste beaucoup à apprendre relativement à cette composante. Elle semble ne pas avoir intégré totalement les différents éléments impliqués. Toutefois, le parcours lui a permis de comprendre que cette composante ne consiste pas uniquement dans le transfert, comme elle semblait le dire lors de la première entrevue, mais qu'elle implique notamment stratégies, démarches et rétroactions, avec lesquelles elle n'est pas encore à l'aise. Dans son cas, il semble que la quantité d'informations et d'exigences que cette composante implique était trop grande pour que le parcours lui ait permis d'augmenter son sentiment d'auto-efficacité. Néanmoins, malgré un certain découragement associé à cela, Chantal semble avoir bénéficié positivement du parcours, qui lui a permis de prendre conscience de ses besoins.

70 Enfin il est intéressant de remarquer qu'en conclusion, Chantal souligne qu'elle n'a pas attendu la fin du parcours pour faire des essais et que sa pratique est déjà en mutation. Le parcours et les vidéos ont donc rapidement stimulé sa pratique et sa volonté de progresser. Il serait pertinent de vérifier maintenant auprès de Chantal si les changements apportés sont plutôt des changements de type 1 ou des changements de 
type 2. Compte tenu de la construction de connaissances plutôt incomplètes et des craintes et difficultés soulevées, la question se pose.

\section{Conclusion}

71 Cette recherche avait pour objectif d'évaluer l'effet du dispositif de formation « Zoom sur l'expertise pédagogique », ou, plus précisément, d'évaluer l'effet d'un parcours de formation créé grâce à ce dispositif et intégrant des exemples de pratiques sur vidéo sur les intentions de changement de pratique d'un groupe d'enseignants du primaire relativement à la compétence professionnelle "Piloter». Elle s'inscrit dans un courant de recherche portant sur l'exploitation de vidéos en formation des enseignants (Colestock \& Sherin, 2009; EdCentric, 2005 ; Galvis \& Nemirovsky, 2003 ; Santagata, 2009 ; Sherin \& van Es, 2009). Comme ces chercheurs, nous avons relevé un certain nombre d'indicateurs qui nous amènent à penser qu'un tel parcours de formation a un effet positif sur le développement professionnel du personnel enseignant et plus précisément sur leurs intentions de changer leur pratique. Nous pouvons tout d'abord retenir que toutes les personnes participantes ont l'intention d'apporter des modifications à leur pratique suite à ce parcours. Même s'il n'est pas toujours possible d'affirmer que les changements qui seront apportés seront des changements de type 2, nous retenons néanmoins que les vidéos donnent envie aux enseignants d'essayer de nouvelles stratégies ou méthodes d'enseignement. Plusieurs des participantes affirment d'ailleurs avoir amorcé, au fur et à mesure du parcours, des essais et des modifications.

72 Nous retenons également que lorsque des vidéos sont perçues comme présentant un idéal impossible à atteindre, le risque de se décourager est présent. Une médiation spécifique devient alors indispensable pour soutenir l'enseignant dans sa démarche. À l'inverse, les vidéos ont validé certains aspects de la pratique des enseignants qui les ont visionnées. Cela a eu pour effet, dans ce cas là, de les rassurer et d'augmenter leur motivation et leur volonté de progresser encore et surtout de changer leur pratique.

Retenons, par ailleurs, l'importance de bien connaître et comprendre la compétence pour être en mesure de déterminer précisément des pistes d'amélioration. En effet, le parcours et les vidéos ont permis aux participants d'affiner leur conception de la compétence et de ses composantes (Meyer, soumis), ce qui leur a permis de mieux définir leurs intentions de changement de pratique. De plus, les personnes ayant défini des intentions précises au préalable semblent retirer beaucoup de ce parcours et semblent mieux cibler ce qu'elles doivent améliorer dans leur pratique.

Retenons, enfin, que les vidéos ont permis aux enseignants de constater à quel point la pratique professionnelle enseignante peut être complexe et ce qu'implique, dans ce contexte, une compétence professionnelle. Ainsi, dans le cas de la composante 4, plusieurs personnes s'estiment moins compétentes après le parcours. Cela ne signifie pas qu'elles soient démotivées ou ne souhaitent pas changer leur pratique: elles comprennent simplement combien il leur reste encore à apprendre et à développer. Cela est très positif, surtout dans le cas d'enseignants qui, comme les participants à cette recherche, présentent, avant même de participer et de suivre le parcours, une volonté de fournir les efforts nécessaires pour apprendre et progresser. Mais il est important de susciter un tel engagement aussi chez les personnes n'ayant pas cette volonté, ou ne jouissant pas de contextes de pratique facilitants ou encore n'ayant pas 
une pratique de référence leur permettant d'élaborer des intentions de changement de pratique pertinent. Il nous semble donc important que les parcours de formation, tels que celui que nous étudions ici, préparent les enseignants à cette prise de conscience, afin qu'ils ne se retrouvent pas devant une montagne qu'ils estiment infranchissable.

La collecte de données de cette recherche s'est déroulée sur une durée d'environ quatre à cinq semaines. Nous n'avons donc pas pu déterminer si les pratiques de ces enseignantes et de ces enseignants avaient véritablement changé. Certes, les données collectées nous ont permis de déceler des intentions de changement de pratique, mais les questions suivantes se posent encore: Quel est le lien entre les intentions de changements de pratique et un changement de pratique effectif? Un tel parcours de formation peut-il avoir un effet sur les changements de pratique des enseignants? Il serait, par exemple, intéressant d'étudier dans quelle mesure ces intentions entraînent des changements significatifs et ce qui peut être modifié dans le parcours ou avec le parcours pour favoriser cela.

Par ailleurs, l'effet vicariant semble devenir important si les enseignants sont en mesure de s'identifier aux enseignants qu'ils observent et d'envisager des possibilités de rencontrer un succès similaire. Derry (2007) propose de travailler à développer des standards qui permettraient de mieux documenter et enrichir les vidéos réalisées. Nous pensons qu'il serait également important d'étudier les différents facteurs facilitant ou compromettant l'identification des enseignants aux personnes observées afin de créer de nouvelles vidéos ou de les accompagner différemment et ainsi d'augmenter davantage l'effet vicariant.

Nous croyons, au terme de cette recherche, que l'utilisation de vidéos d'exemples de pratiques, en particulier lorsque cela est réalisé à l'aide d'un dispositif tel que "Zoom sur l'expertise pédagogique ", peut apporter une contribution importante à l'effort actuellement mis en œuvre pour offrir de nouvelles modalités de formation répondant aux divers besoins actuels du personnel enseignant québécois (Meyer, soumis). Nos recensions nous ont également permis de comprendre qu'il y avait de multiples possibilités d'exploitation des vidéos et qu'il restait encore beaucoup de travail à effectuer pour arriver à en tirer les meilleures possibilités de formation.

\section{BIBLIOGRAPHIE}

Archambault, J., \& Richer, C. (2002). Comment aider les élèves à développer des compétences? Table ronde de personnes-ressources dans le domaine de l'apprentissage. Vie Pédagogique, 123, 18-21.

Bandura, A. (1994). Self-Efficacy. Dans V. S. Ramachaudran (dir.), Encyclopedia of human behavior (Vol. 4) (p. 71-81). New York : Academic Press.

Bandura, A. (1997). Self-Efficacy The Exercise of Control. New York : W.H. Freeman and Company. Bennet, N., \& Carre, C. (dir.). (1993). Learning to teach. London : Routledge. 
Bernard, M. (1999). Penser la mise à distance de la formation. Paris : L'Harmattan.

Bourgeois, E., \& Nizet, J. (1997). Apprentissage et formation des adultes. Paris : Presses universitaires de France.

Brown, J. S., \& Duguid, P. (1991). Organizational learning and communities-of-practice : Toward a unified view of working, learning, and innovation. Organization science, 2(1), 40-57.

Charlier, B. (1998). Apprendre et changer sa pratique d'enseignement. Bruxelles : De Boeck.

Charlier, B., \& Peraya, D. (dir.). (2003). Technologie et innovation en pédagogie. Bruxelles : De Boeck.

Charlier, É. (1998). Former des enseignants professionnels pour une formation continuée articulée à la pratique. Dans L. Paquay, É. Charlier, M. Altet et P. Perrenoud (dir.), Former des enseignants professionnels. Quelles stratégies? Quelles compétences? (p. 97-119). Bruxelle : De Boeck.

Chouinard, R. (2002, mars). Évaluer sans décourager. Communication présentée aux Sessions de formation des personnes-ressources liées à la réforme en éducation, Québec, Québec.

Colestock, A., \& Sherin, M. (2009). Teachers' Sense-Making Strategies While Watching Video of Mathematics Instruction. Journal of Technology and Teacher Education, 17(1), 7-29.

Darling-Hammond, L. (1999). Reshaping Teaching Policy, Preparation, and Practice. Influences of the National Board for Professional Teaching Standards. Washington, DC : American Association of Colleges for Teacher Education, AACTE Publication.

Darling-Hammond, L. (2000a). How Teacher Education Matters. Journal of Teacher Education, 51(3), 166-173.

Darling-Hammond, L. (2000b). Teacher Quality and Student Achievement : A Review of State Policy Evidence. Education Policy Analysis Archives, 8(1). Repéré à http://epaa.asu.edu/ojs/article/ view/392/515

Derry, S. (2007). Video Research in Classroom and Teacher Learning (Standardize That !). Dans R. Goldman, R. Pea, B. Barron et S. Derry (dir.). Video research in the learning sciences (p. 305-320). Mahwah, NJ : Erlbaum.

Edcentric. (2005). Seeing Math Elementary Final Evaluation Report. Repéré à http:// seeingmath.concord.org/evaluation/SeeingMath_Elementary_Final.pdf

Ertmer, P. A., Conklin, D., \& Lewandowski, J. (2002). Using exemplary Models to Increase Preservice Teachers' Ideas and Confidence for Technology Integration. Dans Proceedings of American Educational Research Association Conference, New Orleans, Louisiana.

Faingold, N. (1997). Le rôle de l'enregistrement vidéo dans l'analyse des situations pédagogiques. Dans G. Mottet (dir.), La vidéo-formation. Autres regards, autres pratiques (p. 167-175). Paris :

L'Harmattan.

Fullan, M., \& Hargreaves, A. (1992). Teacher Development and Educational Change. Dans M. Fullan \& A. Hargreaves (dir.), Teacher Development and Educational Change (p. 1-10). Londres : The Falmer Press.

Gabrielli, J.-P. (1989). Peut-on évaluer la formation continue des enseignants? Nantes : CRDP de Nantes.

Galvis, A., \& Nemirovsky, R. (2003). Interactive video case-based TPD programs : Five critical success factors. Dans G. Richards (dir.), Proceedings of World Conference on E-Learning in Corporate, Government, Healthcare, and Higher Education (p. 1575-1582). Chesapeake, VA : AACE. 
Gouvernement du Québec (1999). Orientations pour la formation continue du personnel enseignant. Québec : Ministère de l'Éducation, Gouvernement du Québec.

Gouvernement du Québec (2001). Programme de formation de l'école québécoise. Québec : Ministère de l'Éducation, Gouvernement du Québec.

Knowles, M. (1990). The Adult Learner (4ème ed.). Houston : Gulf Publishing Company.

Korthagen, F. A. J. (1988). The influence of learning orientations on the development of reflective teaching. Dans J. Calderhead (dir.), Teachers' professional learning (p. 35-51). London : The Falmer Press.

Le Boterf, G. (2000). De quel concept de compétence avons-nous besoin ? Dans G. Le Boterf (dir.), Construire les compétences individuelles et collectives. Paris : Éditions d'Organisation.

Lessard-Hébert, M., Goyette, G., \& Boutin, G. (1990). Recherche qualitative : fondements et pratiques. Ottawa : Agence d'Arc.

Mandeville, L. (2002). Apprendre par l'expérience : un modèle applicable à la formation continue. Dans L. Lafortune, C. Deaudelin, D. Martin et P.-A. Doudin (dir.), La formation continue, de la réflexion à l'action (p. 211-235). Sainte-Foy, Québec : Presses de l'Université du Québec.

Martinet, M. A., Raymond, D., \& Gauthier, C. (2001). La formation à l'enseignement. Les orientations. Les compétences professionnelles. (Document officiel). Québec : Ministère de l'Éducation, Gouvernement du Québec.

Meyer, F. (soumis). Effets d'un dispositif de formation exploitant des vidéos d'exemples de pratiques sur l'apprentissage d'enseignants du primaire. DistanceS.

Meyer, F. (2010). Effets d'un dispositif de formation exploitant des vidéos d'exemples de pratiques sur le développement d'une compétence professionnelle chez des enseignants du primaire (Thèse de doctorat). Université de Montréal.

Mottet, G. (1997). La vidéo, outil de construction des compétences professionnelles des enseignants. Dans G. Mottet (dir.), La vidéo-formation. Autres regards, autres pratiques (p. 21-36). Paris : L'Harmattan.

Paquelin, M. (2000). Du dispositif accompagné au dispositif accompagnant. Repéré à http:// membres.lycos.fr/autograf/Dispositif3.htm

Paquette, G. (2002). L'ingénierie pédagogique. Sainte-Foy, Québec : Presses de l'Université du Québec.

Perrenoud, P. (1996). Enseigner : agir dans l'urgence, décider dans l'incertitude. Paris : ESF éditeur.

Perrenoud, P. (1998). Voyage autour des compétences : vers un métier nouveau ? L'Éducateur, 8(98), 22-27.

Perrenoud, P. (1999). Former les enseignants dans des contextes sociaux mouvants : pratique réflexive et implication critique. Communication présentée à Congrès de l'APNED (Association nationale pour la recherche en éducation), Caxambu (Brasil). Repéré à http://www.unige.ch/fapse/ SSE/teachers/perrenoud/php_main/php_1999/1999_26.html

Plakhotnik, M. (2001). InTime : A PT3 Catalyst Grant. Dans Proceedings of Society for Information Technology and Teacher Education International Conference 2001, Orlando, Florida.

Projet Polifemo. (2001). Démarche d'ingénierie des dispositifs de formation ouverte. Fonds Social Européen. Repéré à http://www.eduter-cnpr.fr/pdf/pttbiblio/guid08N1.pdf 
Russell, T., \& McPherson, S. (2001). Indicators of success in teacher education. Communication présentée à Pan-Canadian Education Research Agenda Symposium Teacher Education/Educator Training: Current Trends and Future Directions, Université Laval, Québec. Repéré à http:// www.cesc.ca/pceradocs/2001/papers/01Russell_McPherson_e.pdf

Santagata, R. (2009). Designing Video-Based Professional Development for Mathematics Teachers in Low-Performing Schools. Journal of Teacher Education. Theme Issue : Innovative Uses of Technology in Teacher Education, 60(1), 38-51.

Schön, D.A. (1994). Le praticien réflexif - À la recherche du savoir caché dans l'agir professionnel. Montréal : Les Éditions Logiques.

Sherin, M.G., \& van Es, E.A. (2009). Effects of Video Club Participation on Teachers' Professional Vision. Journal of Teacher Education, 60, 20-37.

Shulman, L. S. (1986). Those who understand, knowledge growth in teaching. Educational research, 15(2), 4-14.

Tschannen-Moran, M., Woolfolk Hoy, A., \& Hoy, W. K. (1998). Teacher Efficacy : its Meaning and Measure. Review of Educational Research, 68(2), 202-248.

Watzlawick, P., Weakland, J., \& Fisch, R. (1975). Changements : Paradoxes et psychothérapie. Paris : Éditions du Seuil.

\section{ANNEXES}

Annexe $1:$ La compétence et les composantes ciblées

Afin de rendre cette recherche plus précise et plus efficace, nous avons décidé de nous concentrer sur la compétence 4 du référentiel publié par le MELS : «Piloter des situations d'enseignement-apprentissage pour les contenus à faire apprendre, et ce, en fonction des élèves concernés et du développement des compétences visées dans le programme de formation » (Martinet et al., 2001, p. 85).

Cette compétence se découpe, selon la définition proposée par le MELS, en cinq composantes :

"Créer des conditions pour que les élèves s'engagent dans des situations-problèmes, des tâches ou des projets significatifs en tenant compte de leurs caractéristiques cognitives, affectives et sociales » (p. 86) ;

« Mettre à disposition des élèves les ressources nécessaires à la réalisation des situations d'apprentissage proposées » (p. 87);

«Guider les élèves dans la sélection, l'interprétation et la compréhension de l'information disponible dans les diverses ressources ainsi que dans la compréhension des éléments des situations-problèmes ou des exigences d'une tâche ou d'un projet » (p. 87) ;

« Encadrer les apprentissages des élèves par des stratégies, des démarches, des questions et des rétroactions fréquentes et pertinentes de manière à favoriser l'intégration et le transfert des apprentissages » (p. 87);

« Habiliter les élèves à travailler en coopération » (p. 88). 
La cinquième composante n'a pas été retenue dans le cadre de cette recherche car elle n'était pas abordée dans la formation en question.

\section{RÉSUMÉS}

Pour soutenir le développement professionnel des enseignants québécois, un dispositif de formation en ligne intégrant des vidéos de pratique, appelé «Zoom sur l'expertise pédagogique », a été développé. Il propose de nouvelles stratégies de formation basées principalement sur l'observation et l'analyse de ces vidéos ainsi que divers outils stimulant la réflexion sur la pratique.

Cette recherche avait pour objectif d'évaluer l'effet d'un parcours de formation créé grâce à ce dispositif et intégrant des exemples de pratiques sur vidéo sur les intentions de changement de pratique d'un groupe d'enseignants du primaire relativement à la compétence professionnelle «Piloter des situations d'enseignement-apprentissage ». Nous avons pu confirmer, à l'aide d'un modèle d'analyse complexe, l'effet positif, dans certaines conditions, sur ces intentions en identifiant notamment des effets sur le degré de confiance de ces enseignants et leur capacité à valider leur propre pratique.

To support the professional development of teachers in Quebec, a web-based professional teacher development application called "Zooming in on Teaching Expertise" was developed. It offers new training strategies based primarily on observation and analysis of videos of practice as well as various tools for promoting reflection on practice.

Our aim was to evaluate the effect of a training course entailing examples of practice on video and created within the application. In particular, the study focused on participating elementary school teachers' intentions to modify their practice with respect to the professional competency : "To pilot teaching/learning situations". Using a complex theoretical framework, we confirmed the positive effect, under certain conditions, on teachers' intentions through identifying particular effects on their self-confidence and their ability to validate their own practice.

\section{INDEX}

Mots-clés : changement de pratique, compétence professionnelle, développement professionnel, dispositif de formation, multimédia, vidéo de pratiques

\section{AUTEUR}

\section{FLORIAN MEYER}

Université de Sherbrooke

Faculté d'éducation, Département de Pédagogie, Bureau A2-237

2500, Boulevard de l'Université, Sherbrooke (QC), J1K 2R1

Tel. : +1 8198218000 | poste 63089

florian.meyer@usherbrooke.ca 